\title{
Preoperative computerized tomography screening for COVID-19 pneumonia in asymptomatic patients: experiences from two centers
}

\author{
Terman Gümüs ${ }^{1} \cdot$ Zeynep Unal Kabaoglu ${ }^{2}$ - Bilgen Coskun ${ }^{2} \cdot$ Furkan Kartal $^{1} \cdot$ Feyzi Artukoglu $^{3}$. \\ Kayhan Cetin Atasoy ${ }^{1}$
}

Received: 5 August 2020 / Accepted: 16 October 2020 / Published online: 31 October 2020

(c) Japan Radiological Society 2020

\begin{abstract}
Purpose The aim of this retrospective study is to evaluate the preoperative screening performance of chest CT (computerized tomography) examination to detect COVID-19 positive individuals.

Materials and methods In this retrospective study 218 adult patients who had preoperative chest CT and RT-PCR were enrolled. CT imaging results, which have been reported according to the Radiological Society of North America expert consensus on COVID-19, were collected from the picture archiving and communicating system. Demographic data, planned surgeries, and postoperative outcomes were collected from the electronic patient records.

Results One patient (0.5\%) showed typical CT features for COVID-19 pneumonia; 12 patients (5.5\%) were reported as indeterminate, and eight (3.7\%) were reported as atypical for COVID-19 pneumonia. Only one of the three patients with positive RT-PCR had abnormalities on CT. When RT-PCR tests were taken as reference, the sensitivity, specificity, and accuracy of chest CT in showing COVID-19 infection in asymptomatic patients were 33.3\%, 90.7\%, and 90.0\%, respectively. Conclusion Chest CT screening for COVID-19 has a very low yield in asymptomatic preoperative patients and shows falsepositive findings in $9.2 \%$ of cases, potentially leading to unnecessary postponing of the surgery.
\end{abstract}

Keywords COVID- $19 \cdot$ Chest CT $\cdot$ Screening

\section{Introduction}

Since the first case reported in China on December 31, 2019, COVID-19 pandemic has rapidly spread worldwide, resulting in more than 10 million of confirmed cases and half million deaths in $<6$ months $[1,2]$. Healthcare workers are most vulnerable; indeed, up to $20 \%$ of responding health care providers were reported to be infected [3]. Although the final diagnosis rests with reverse-transcriptase polymerase chain reaction (RT-PCR), this test has several shortcomings including limited availability, relatively long turnaround times, and a low sensitivity varying between 56 and 83\% [4].

Terman Gümüs

tgumus@kuh.ku.edu.tr

1 Department of Radiology, University of Koc School of Medicine, Davutpaşa Caddesi No. 4, Topkapı, 34010 Istanbul, Turkey

2 Radiology Department, American Hospital, Istanbul, Turkey

3 Anesthesiology and Reanimation Department, American Hospital, Istanbul, Turkey
Patients with COVID-19 disease who undergo surgery suffer a complicated postoperative course [5, 6]. Studies have shown that up to $51.2 \%$ of these patients had pulmonary complications and among patients who had pulmonary complications after surgery 30 -day mortality rate was $38 \%$ [7]. Also, these patients increase the risk of disease transmission to other patients in the hospital and health care personnel [8].

Aforementioned diagnostic difficulties associated with the molecular methods coupled with dismal postoperative course and risk of cross-infection to others have led medical care providers to search for complementary diagnostic tools $[9,10]$. Many studies have demonstrated a higher sensitivity of chest CT for COVID-19 pneumonia than RT-PCR [11-13]. CT was also shown to be positive in a subset of asymptomatic COVID-19 patients [14, 15]. Thus, CT has been used in adjunct to molecular methods in asymptomatic patients scheduled for surgery to screen for COVID19 disease.

There is very limited knowledge regarding the use of CT in this peculiar setting. We, therefore, aimed to evaluate the 
results of chest CT screening for COVID-19 pneumonia in asymptomatic patients before surgery.

\section{Materials and methods}

\section{Patients}

The study was a retrospective analysis and approved by the Ethics Committee of Koc University Faculty of Medicine, which waived the requirement for patients' informed consent. Between April 20, 2020 and May 31, 2020218 adult patients who admitted to two hospitals (one university hospital and one private hospital from the same city) for surgery were enrolled. All patients had undergone a preoperative chest CT and RT-PCR test. Patients who had symptoms suggesting COVID-19 infection were excluded. Patients' demographic characteristics, including the age and gender, and type of surgery were recorded (Table 1).

\section{Image acquisition}

CT scans were acquired using multidetector CT scanners with 64 (Somatom Definition AS) or 128 (Somatom Definition Flash) detector rows (Siemens Healthineers, Forchheim, Germany) with patients in the supine position. The entire chest starting from the lung apices down to posterior costophrenic sulci was scanned with $0.625 \mathrm{~mm}$ collimation, 80-120 kVp, and 20-150 mAs. Images were reconstructed with a slice thickness of $1-1.5 \mathrm{~mm}$ and an interval of $1 \mathrm{~mm}$. No intravenous contrast medium was administered.

\section{Data collection}

Chest $\mathrm{CT}$ images were reported by six radiologists in two centers with 6-20 years of experience in thoracic imaging.

Table 1 Distribution of patients on the basis of intervention

\begin{tabular}{ll}
\hline Intervention & Patients $(n=218)$ \\
\hline General surgery & $59(27.0 \%)$ \\
Neurosurgery & $38(17.4 \%)$ \\
Orthopedics and traumatology & $27(12.3 \%)$ \\
Obstetrics and gynecology & $19(8.7 \%)$ \\
Urosurgery & $17(7.8 \%)$ \\
Thoracic surgery & $15(6.9 \%)$ \\
Cardiovascular surgery & $7(3.2 \%)$ \\
Ear nose and throat surgery & $7(3.2 \%)$ \\
Plastic and reconstructive surgery & $4(1.8 \%)$ \\
Ophthalmology & $2(0.9 \%)$ \\
Interventional procedures (gastrointestinal endos- & $23(10.6 \%)$ \\
\multicolumn{2}{c}{ copy, bronchoscopy, cardiac catheterization) } \\
\hline
\end{tabular}

All CT reports and CT dose information were retrospectively collected from the picture archive and communication system (PACS). CT findings were classified according to the Radiological Society of North America (RSNA) chest CT classification system for reporting COVID-19 pneumonia as follows: (a) CT imaging features typical, (b) indeterminate, and (c) atypical for COVID-19 pneumonia, and (d) No CT features to suggest pneumonia [16].

Typical CT imaging features included peripheral, bilateral ground-glass opacities (GGO) with or without consolidation or crazy-paving appearance, multifocal GGO of rounded morphology with or without consolidation or crazy-paving, and reverse halo sign or other findings of organizing pneumonia (Fig. 1).

Indeterminate findings included cases who lacked the typical features but displayed multifocal, diffuse, perihilar, or unilateral GGO with or without consolidation lacking a specific distribution and are non-rounded or non-peripheral. Few very small GGO with a non-rounded and non-peripheral distribution were also regarded indeterminate (Fig. 2).

Atypical cases lacked the typical or indeterminate features but had isolated lobar or segmental consolidation without GGO, or discrete small nodules (centrilobular, "treein-bud"), lung cavitation, and smooth interlobular septal thickening with pleural effusion (Fig. 3).

Additionally reports were searched for incidental malignant findings.

\section{Statistical analysis}

Statistical analyses were performed using IBM SPSS Statistics for Windows, version 24 (IBM Corp., Armonk, NY, USA). Measurement data are were expressed as mean \pm standard deviation, and categorical variables were reported as counts and percentages.

\section{Results}

\section{Demographic characteristics and general information}

218 patients [107 patients from the university hospital and 111 patients from the private hospital) were enrolled in this study [114 male, 104 female, mean age $56 \pm 15.6$ years (range 24-91 years)]. Mean DLP value for university hospital was $200.14 \mathrm{mGy} \times \mathrm{cm}$ and for the private hospital was $214.07 \mathrm{mGy} \times \mathrm{cm}$.

Of the whole study group, 197 patients $(90.4 \%)$ had no CT findings suggesting pneumonia. One patient $(0.5 \%)$ showed typical CT features for COVID-19 pneumonia; 12 patients $(5.5 \%)$ were reported as indeterminate, and eight (3.7\%) were reported as atypical for COVID-19 
Fig. 1 a, b An example for a patient reported as a typical pattern for COVID-19. Axial chest CT showing (a) bilateral GGO in upper lobes and (b) in left lower lobe
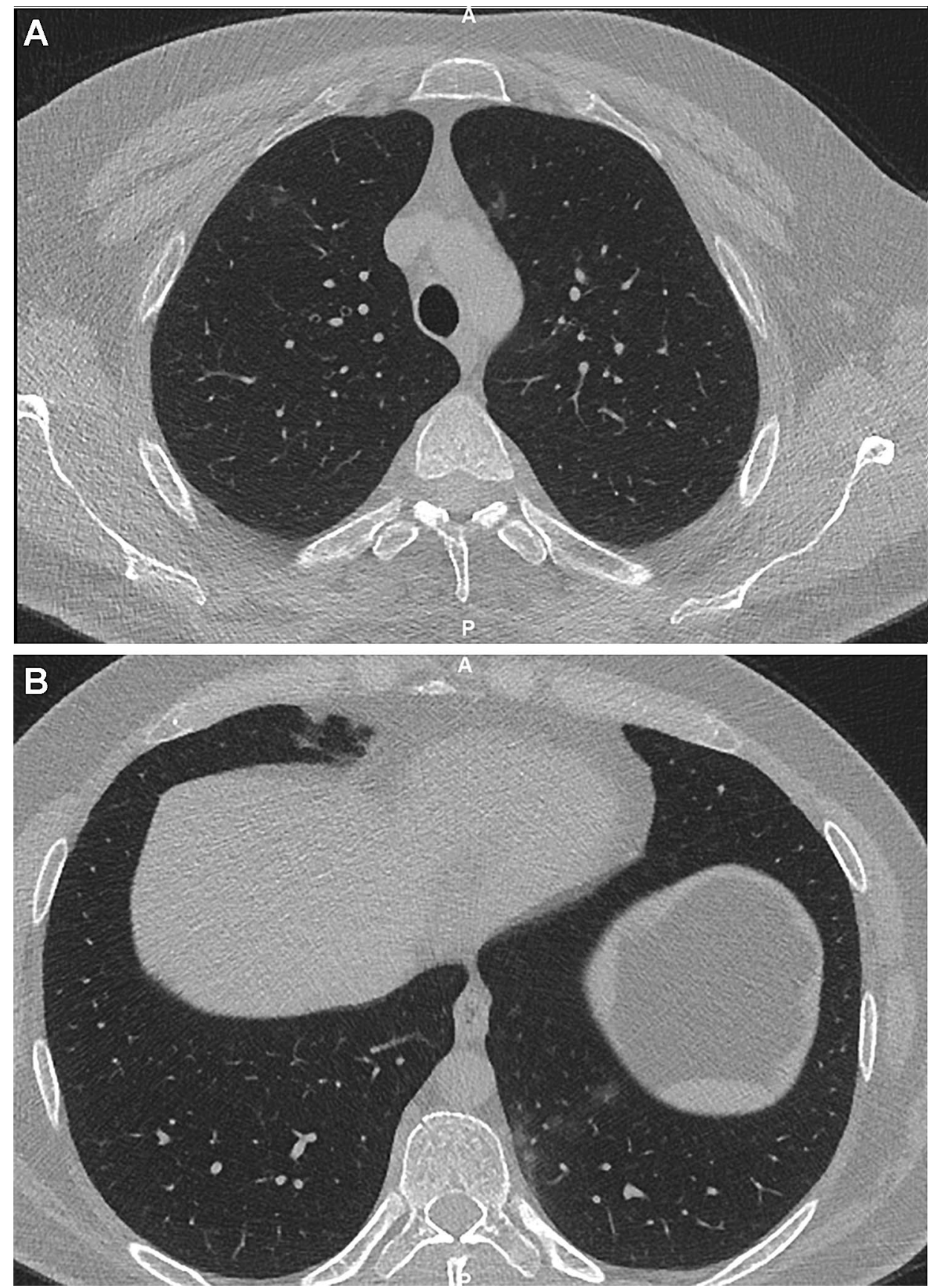

pneumonia. None of the patients had incidentally discovered thoracic malignancies or newly discovered metastasis.

Of 218 patients, three $(1.4 \%)$ had positive and 215 (98.6\%) had negative RT-PCR test result. Only one of the three patients with positive RT-PCR had findings on chest CT that may be attributed to pneumonia. Of 215 patients with negative RT-PCR result, 20 (9.3\%) had CT abnormalities attributable to pneumonia. 12 of these 20 patients had indeterminate CT findings and eight had atypical CT findings for COVID-19.

\section{Performance of chest CT-screening in diagnosing COVID-19 in asymptomatic patients}

In 21 patients COVID-19 pneumonia could not be ruled out based on chest CT findings. Only one of these had typical CT findings for COVID 19 pneumonia whereas the rest had indeterminate or atypical CT findings. When RT-PCR tests were taken as reference, the sensitivity, specificity, and accuracy of chest CT in showing COVID19 disease were $33.3 \%, 90.7 \%$, and $90.0 \%$, respectively. 
Fig. 2 An example for a patient reported as an indeterminate pattern for COVID-19. Axial chest CT showing focal GGO confined to the left upper lobe
Fig. 3 An example for a patient reported as an atypical pattern for COVID-19. Axial chest CT showing discrete centrilobular small nodules in the left lower lobe. Other lung lobes were normal
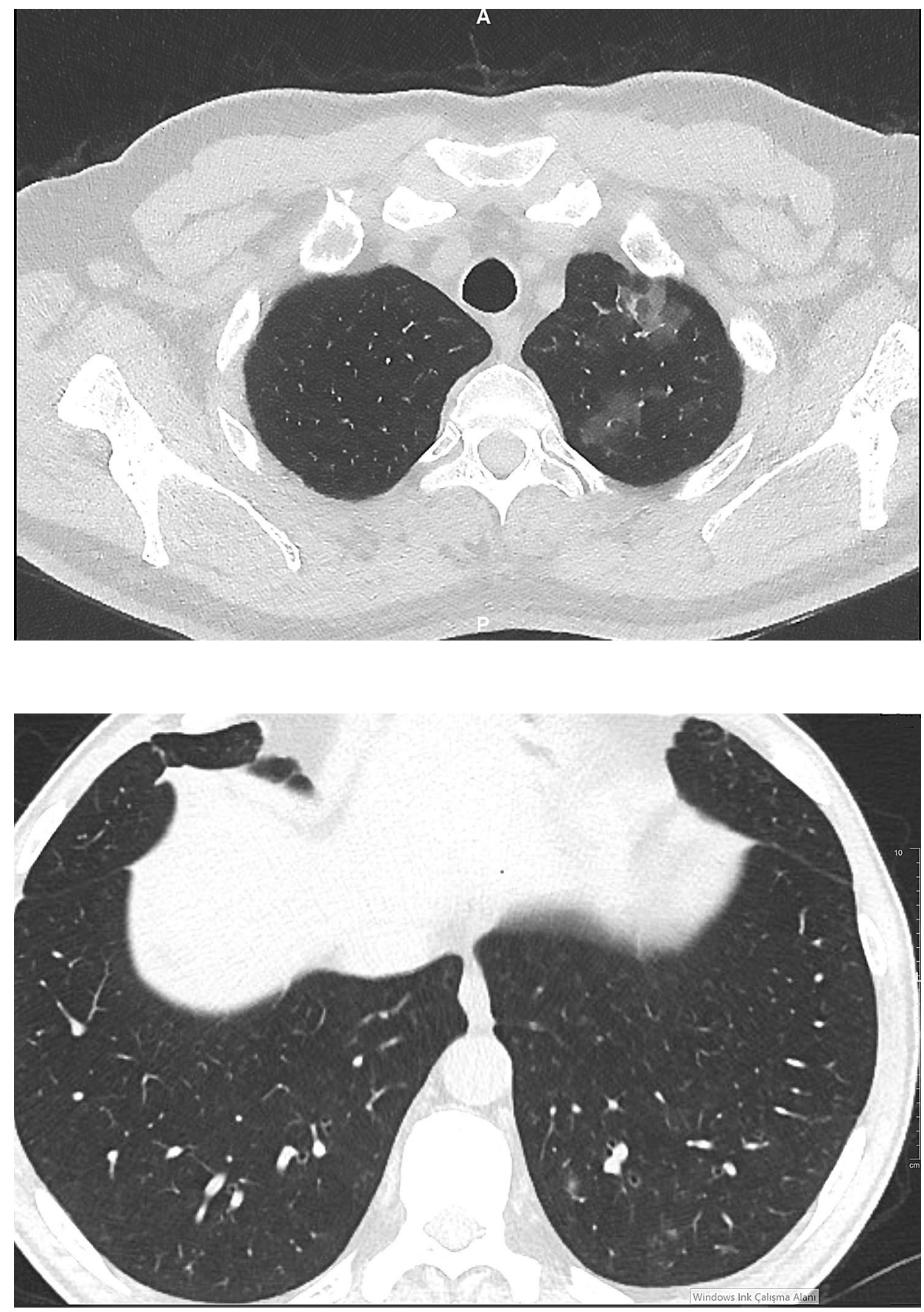

The positive and negative predictive values were $4.8 \%$ and $99.0 \%$, respectively.

Two patients with positive RT-PCR results had no lesions suggesting COVID-19 pneumonia on chest CT. All 12 patients with indeterminate CT findings and all eight patients with atypical CT findings for COVID-19 were RT-PCR negative. Of the 20 patients with indeterminate or atypical findings, $16(80 \%)$ had unilateral lung lesions, 12 (60\%) had unilateral ground-glass opacities, two (10\%) had segmental/subsegmental consolidation, 4
(20\%) had unilateral discrete small nodules, and two (10\%) had smooth interlobular septal thickening.

$72(33.0 \%)$ Patients needed emergent and 146 (67.0\%) patients needed elective surgery. All but three patients who needed emergent surgery had negative chest CT and RTPCR results. Three patients had indeterminate $\mathrm{CT}$ findings and were operated without delay with relevant precautions for airborne infection. RT-PCR tests repeated after surgery in these three patients were also normal. 
Out of the 146 patients who needed elective surgery, 126 had negative CT and PCR results and underwent surgery timely. Nine patients with indeterminate $\mathrm{CT}$ and eight patients with atypical CT findings had negative PCR results. 15 of these patients were operated without a delay. In the remaining two patients, both of whom had atypical CT findings, surgery was delayed for 2 and 3 days, after a repeat RT-PCR was negative.

One patient who had typical CT findings and a positive RT-PCR test was operated after 24 days of treatment with Plaquenil (Fig. 2).

None of the 21 patients with positive chest CT findings suffered from postoperative complications and were discharged with a mean hospital stay of 5.1 days.

Two patients had negative CT findings but positive PCR results. One of the patients had elective endoscopy and the other lumbar discectomy. Both of the interventions were canceled till a negative PCR result is obtained. Both patients were under surveillance without medication administration.

\section{Discussion}

In this study, we investigated the performance of chest CT as a screening method for COVID-19 in asymptomatic surgery patients. In our study, CT was true positive in one patient, whereas it was false positive in 20 patients and $10 \%$ of these patients encountered an unnecessarily delay for surgery. All patients who faced with a delay were candidates for elective surgeries, none of the emergency cohort requiring surgery encountered a delay. None of these 20 patients experienced an unexpected poor postoperative course. These findings support the previously reported low specificity of chest CT for COVID-19 pneumonia [12].

On the other hand in our study CT showed features of pneumonia in only one of three asymptomatic COVID-19 positive patients. The positivity rate is lower than reported on an earlier study, in which CT was positive in $79 \%$ of asymptomatic test-positive patients [17]. However, that study was performed on passengers in a cruise ship under quarantine, who had a longer close contact with COVID-19 patients. According to a metanalysis the pooled estimate of the overall rate of initially asymptomatic cases with positive chest CT findings was 63\% [18]. In the literature, there are different diagnostic sensitivity rates of chest $\mathrm{CT}$ at detecting COVID-19 in asymptomatic individuals. This is most probably because of the heterogeneity of study groups; on one side cruise ship passengers with close contact under quarantine, on the other side preoperative patients without a known contact history. Another factor that may underlie the rate of positivity of chest $\mathrm{CT}$ in asymptomatic patients may be the prevalence of infection in the community, i.e. more asymptomatic patients may be expected to have $\mathrm{CT}$ findings of pneumonia when the prevalence and transmission rate of infection is high. The temporal span of the study coincides to a time period when the first peak of the epidemic curve has been over and the curve started to bend downwards, with a mean daily confirmed cases of 1873 in Turkey [19].

An important finding of our study is that CT shows findings attributable to pneumonia in $9.6 \%$ of asymptomatic preoperative patients. Furthermore, these findings were typical or indeterminate for COVID-19 pneumonia in 5.9\% of patients. Our findings are similar to those of Chetan et al. who reported that $3 \%$ of preoperative patients had classic/ probable and $4 \%$ had indeterminate findings for COVID-19 pneumonia [20].

Limitations of our study involve its retrospective design with a heterogenous preoperative patient group including candidates for emergency and elective surgery.

This retrospective study showed that CT screening in asymptomatic cases is not adequately sensitive in detecting RT-PCR positive COVID-19 patients. Moreover, an important minority of patients may show false-positive findings, which may potentially mislead patient management, including unnecessary postponing of the interventions. Besides potential harms of radiation must be kept in mind while considering chest $\mathrm{CT}$ as a screening method. Preoperative CT screening should not be ordered on a routine basis in asymptomatic patients, especially when rapid RT-PCR tests are available.

\section{Compliance with ethical standards}

Conflict of interest The author(s) declare(s) that there is no conflict of interest.

\section{References}

1. World Health Organization. WHO Timeline-COVID-19. Available at: https://www.who.int/news-room/detail/27-04-2020-whotimeline---COVID-19. Accessed 7 Jul 2020.

2. World Health Organization. WHO Coronavirus Disease (COVID19) Available at: https://covid19.who.int/. Accessed July 7, 2020.

3. Remuzzi A, Remuzzi G. COVID-19 and Italy: what next? Lancet. 2020;395(10231):1225-8. https://doi.org/10.1016/S0140 $-6736(20) 30627-9$.

4. Kokkinakis I, Selby K, Favrat B, et al. Performance du frottis nasopharyngé-PCR pour le diagnostic du Covid-19-recommandations pratiques sur la base des premières données scientifiques [Covid-19 diagnosis: clinical recommendations and performance of nasopharyngeal swab-PCR]. Rev Med Suisse. 2020;16(689):699-701.

5. Nahshon C, Bitterman A, Haddad R, et al. Hazardous postoperative outcomes of unexpected COVID-19 infected patients: a call for global consideration of sampling all asymptomatic patients before surgical treatment. World J Surg. 2020;44:2477-81. https ://doi.org/10.1007/s00268-020-05575-2. 
6. Rescigno G, Firstenberg M, Rudez I, et al. A case of postoperative Covid-19 infection after cardiac surgery: lessons learned. Heart Surg Forum. 2020;23(2):E231-3. https://doi.org/10.1532/ hsf.3011.

7. COVID Surg Collaborative. Mortality and pulmonary complications in patients undergoing surgery with perioperative SARS-CoV-2 infection: an international cohort study. Lancet. 2020;396(10243):27-38. https://doi.org/10.1016/S0140 -6736(20)31182-X.

8. Heinzerling A, Stuckey MJ, Scheuer T, et al. Transmission of COVID-19 to health care personnel during exposures to a hospitalized patient-Solano County, California, February 2020. MMWR Morb Mortal Wkly Rep. 2020;69(15):472-6. https:// doi.org/10.15585/mmwr.mm6915e5.

9. Kimball A, Hatfield KM, Arons M, et al. Public Health-Seattle \& King County; CDC COVID-19 Investigation Team. Asymptomatic and presymptomatic SARS-CoV-2 infections in residents of a long-term care skilled nursing facility: King County, Washington March 2020. MMWR Morb Mortal Wkly Rep. 2020;69:377.

10. Day M. COVID-19: four fifths of cases are asymptomatic. China Fig Indic BMJ. 2020;369:1375.

11. Fang Y, Zhang H, Xie J, et al. Sensitivity of chest CT for COVID19: comparison to RT-PCR. Radiology. 2020;19:200432.

12. Ai T, Yang Z, Hou H, et al. Correlation of chest CT and RTPCR testing in coronavirus disease 2019 (COVID-19) in China: a report of 1014 cases. Radiology. 2020;296:E32-40. https://doi. org/10.1148/radiol.2020200642.

13. Long $\mathrm{C}, \mathrm{Xu} \mathrm{H}$, Shen Q, et al. Diagnosis of the coronavirus disease (COVID-19): rRT-PCR or CT? Eur J Radiol. 2020;126:108961. https://doi.org/10.1016/j.ejrad.2020.108961.

14. Yoshimura Y, Sasaki H, Horiuchi H, et al. Clinical characteristics of the coronavirus disease 2019 (COVID-19) outbreak on a cruise ship. J Infect Chemother. 2020;26:1177-80. https://doi. org/10.1016/j.jiac.2020.06.010.
15. Long QX, Tang XJ, Shi QL, et al. Clinical and immunological assessment of asymptomatic SARS-CoV-2 infections. Nat Med. 2020. https://doi.org/10.1038/s41591-020-09656.

16. Simpson S, Kay FU, Abbara S, et al. Radiological Society of North America expert consensus statement on reporting chest CT findings related to COVID-19 endorsed by the Society of Thoracic Radiology, the American College of Radiology, and RSNA-Secondary Publication. J Thorac Imaging. 2020;35(4):219-27. https ://doi.org/10.1097/RTI.0000000000000524.

17. Bernheim A, Mei X, Huang M, Yang Y, Fayad ZA, Zhang N, Diao K, Lin B, Zhu X, Li K, Li S, Shan H, Jacobi A, Chung M. Chest CT findings in coronavirus disease-19 (COVID-19): relationship to duration of infection. Radiology. 2020;295(3):200463. https:// doi.org/10.1148/radiol.2020200463.

18. Tsikala-Vafea M, Atalla E, Kalligeros M, Mylona E, Shehadeh $\mathrm{F}$, Mylonakis E. Chest CT findings in asymptomatic cases with COVID-19: a systematic review and meta-analysis. Clin Radiol. 2020. https://doi.org/10.1016/j.crad.2020.07.025.

19. Recent coronavirus status in Turkey, Ministry of Health Turkey. https://covid19.saglik.gov.tr. Published June 20, 2020. Accessed 23 Sept 2020.

20. Chetan MR, Tsakok MT, Shaw R, et al. Chest CT screening for COVID-19 in elective and emergency surgical patients: experience from a UK tertiary centre. Clin Radiol. 2020;75(8):599-605. https://doi.org/10.1016/j.crad.2020.06.006.

Publisher's Note Springer Nature remains neutral with regard to jurisdictional claims in published maps and institutional affiliations. 